\title{
A comparative study of independent particle model based approaches for thermal averages
}

\author{
SUBRATA BANIK*, TAPTA KANCHAN ROY* and M DURGA PRASAD* \\ School of Chemistry, University of Hyderabad, Hyderabad 500 046, India \\ e-mail: subratachem@gmail.com; taptakanchan@gmail.com; mdpsc@uohyd.ernet.in
}

MS received 13 October 2012; revised 20 May 2013; accepted 27 May 2013

\begin{abstract}
A comparative study is done on thermal average calculation by using the state specific vibrational self-consistent field method (ss-VSCF), the virtual vibrational self-consistent field (v-VSCF) method and the thermal self-consistent field (t-SCF) method. The different thermodynamic properties and expectation values are calculated using these three methods and the results are compared with full configuration interaction method (FVCI). We find that among these three independent particle model based methods, the ss-VSCF method provides most accurate results in the thermal averages followed by t-SCF and the v-VSCF is the least accurate. However, the ss-VSCF is found to be computationally very expensive for the large molecules. The t-SCF gives better accuracy compared to the V-VSCF counterpart especially at higher temperatures.
\end{abstract}

Keywords. Self-consistent field theory; partition function; density matrix.

\section{Introduction}

The canonical partition functions $Z(T)$ plays an important role in statistical mechanics. The relation between different thermodynamic quantities such as the free energy, internal energy or entropy to the partition function is well-established. The partition function of a molecule is defined as

$$
Z(T)=\sum_{n} e^{-\varepsilon_{n} / k_{B} T}
$$

Here, $\varepsilon_{n}$ is the energy eigenvalue of $n$th state, $k_{B}$ is the Boltzmann's constant and $T$ is the temperature. Thus, one requires to evaluate the complete set of molecular energy eigenvalues and sum up the associated Boltzmann's factors to calculate the molecular partition function at a particular temperature. Once the partition function is calculated, the thermodynamic properties are easily obtained by using the standard statistical mechanical equations. For example,

$$
\begin{gathered}
A(T)=k_{B} T \ln Z(T), \\
U(T)=U(0)+\frac{\sum_{n} \varepsilon_{n} \varepsilon^{-\varepsilon_{n} / k_{B} T}}{Z(T)}, \\
S(T)=k_{B} \ln Z(T)+\frac{U(T)}{T} .
\end{gathered}
$$

Here, $A$ is the Helmholtz free energy, $U$ is the internal energy, $U(0)$ is the zero point energy and $S$ is the entropy.

\footnotetext{
*For correspondence
}

With the development of quantum chemical methods over last few decades, the accurate descriptions of molecular energy levels are achievable. The calculation of the molecular energy levels is carried out by invoking a series of approximations to calculate thermal averages. The nuclear and electronic motions are separated by invoking the Born-Oppenheimer approximation at the first stage. Mostly the ground electronic state alone is considered to calculate the partition functions due to the large separation of the electronic states in a molecule. The potential energy surface associated with this ground electronic state is used to calculate the rovibrational energy levels. The rotational and the vibrational motions are often separated by using the rigid rotor model for the rotational degrees of freedom. The rotational partition functions for the rigid rotors are analytically available. The remaining vibrational problem is often solved in the harmonic oscillator (HO) approximation, where the analytical solution to the partition function is available in terms of the normal mode frequencies.

The harmonic approximation provides reasonable description of the vibrational motion of semi-rigid molecules for the lower lying vibrational states and hence it accounts for reasonably accurate results for the thermodynamic properties of such molecules, especially in the low temperature domain. The partition functions of semi-rigid molecules at lower temperature are dominated by Boltzmann's factors of the lower lying vibrational states. However, the higher vibrational states are affected by the anharmonicity to a greater 
extent. With the increase of temperature, the partition functions of molecules are influenced by the Boltzmann's factors of the higher vibrational states. Hence, the HO approximation often fails to provide an accurate description of the thermal averages of molecular properties at higher temperatures. Several approaches have been discussed in literature to incorporate such anharmonic effects. ${ }^{1-28}$

One of the most common ways to describe the anharmonicity in the molecular vibrations is the use of quartic potential energy surface (PES) in normal coordinates. Several studies have been done to test the reliability of using the $a b$ initio quartic force fields for the calculation of thermal properties. ${ }^{1-3}$ The vibrational Hamiltonian with such quartic potential is a many-body Hamiltonian and hence the exact analytical solution of the associated Schrödinger equation is not possible. Several authors used the vibrational configuration interaction (VCI) method to calculate the thermal properties of molecules. ${ }^{20,26}$ In this method, one constructs the vibrational anharmonic Hamiltonian in a chosen basis and diagonalizes it to obtain the vibrational state energies. The VCI is conceptually simple and gives exact results within the chosen basis. However, the dimension of VCI matrix increases exponentially with the number of vibrational degrees of freedom. This makes the VCI method practically inapplicable for large molecules. Such limitation of VCI provides motivations to formulate alternative methods and approximations to study the anharmonic vibrational problem or partition functions directly.

The simplest and most common approximation to solve the many-body problems in quantum and statistical mechanics is the mean field approximation or the independent particle model (IPM). Here, the complicated many-body potential is replaced by averaged sum of the one body potentials. The vibrational selfconsistent field (VSCF) ${ }^{29-33}$ method is such a mean field approach to solve the Schrödinger equation associated with the molecular anharmonic vibrations. A few approaches have been developed to calculate the thermal averages of molecular properties based on the VSCF methodology. One is the state-specific vibrational self-consistent field (ss-VSCF) approach. ${ }^{27}$ Here, the single particle potential is optimized for each vibrational state and VSCF calculation is done for each state. Thus a total $N$ number of VSCF calculations are performed to get $N$ vibrational state energies and Eq. (1) is used to calculate the partition function. The VSCF calculation for each state scales linearly with the vibrational degrees of freedom and thus avoids the dimensionality problem of the VCI method. However, the sum-over-states required for the partition function still remains in the ss-VSCF method for the partition functions. In a calculation for a molecule with $D$ degrees of freedom and $B$ single particle functions for each degree of freedom, the total number of states is $B^{D}$. Thus, the second part of the calculation still scales exponentially with the number of degrees of freedom. Moreover, the VSCF method often suffers from convergence problem, especially for higher states. This makes the ss-VSCF method often inaccessible for large molecules. Hansen et al. considered a further approximation in the VSCF method to overcome this bottleneck of sum-over-states problem. This method is known as virtual vibrational self-consistent field method (v-VSCF). ${ }^{27}$ Here, one performs the VSCF calculation only for the ground vibrational state. The virtual modal energy differences of this vibrational ground state calculation are taken as the excited states energies. The partition function and other thermal averages are calculated in usual manner. The v-VSCF method showed reasonable accuracy for the thermal properties calculations at lower temperatures. ${ }^{27}$ Because it requires only one VSCF calculation, this approximation is not subject to the exponential bottleneck.

Recently, Roy and Prasad ${ }^{28}$ proposed an alternative method to calculate the thermal averages that uses Feynman variational principle ${ }^{34}$ based on the GibbsBogoliubov inequality. Here, a separable ansatz of thermal density matrix based on the IPM is used to calculate the thermal averages. The resulting theory resembles the VSCF procedure with the replacement of quantum mechanical averages with their thermal counterpart. For this reason, this method is termed as thermal self-consistent field theory (t-SCF). The computational effort of this approach for a given temperature is similar to a single VSCF calculation.

The goal of the present work is to make a systematic comparison of these three methods based on IPM, i.e., the ss-VSCF, v-VSCF and t-SCF to calculate the thermal averages at a given temperature. All the three methods are based on the IPM but, the working equations are obtained by different approaches, and hence differ from each other. Different aspects of these three methods are analysed and their performances are compared based on the computational difficulties and accuracy in the thermal average calculations. The accuracy in the calculation of thermal averages by these methods is compared with full VCI (FVCI) values. The performance of these three methods is compared over a range of thermal properties and a wide range of temperature starting from $10 \mathrm{~K}$ to $1000 \mathrm{~K}$. The paper is organized as follows. In the next section, we give brief review of theoretical aspects of these three methods. We calculate different thermal properties of $\mathrm{Cl}_{2} \mathrm{O}$ and $\mathrm{O}_{3}$ molecules by these 
three methods and compare them with the results of the FVCI method. The results are presented in section 3. Finally, some concluding remarks are given in section 4.

\section{Theory}

\subsection{Preliminaries}

Within the Born-Oppenheimer approximation in normal coordinate the Watson Hamiltonian for the rovibrational motion of a molecule is given by ${ }^{35}$

$$
H=\sum_{i}-\frac{p_{i}^{2}}{2}+V(Q)+V_{c}+V_{W}
$$

Here, $Q_{i}$ and $P_{i}$ represent the mass-weighted normal coordinates and their conjugate momenta. $V(Q)$ is the potential energy function. This is often approximated by a quartic polynomial,

$$
\begin{aligned}
V= & \sum_{i} f_{i i} Q_{i}^{2}+\sum_{i \leq j \leq k} f_{i j k} Q_{i} Q_{j} Q_{k} \\
& +\sum_{i \leq j \leq k \leq l} f_{i j k l} Q_{i} Q_{j} Q_{k} Q_{l} .
\end{aligned}
$$

The coefficients $\left\{f_{i i}, f_{i j k}, f_{i j k l}\right\}$ are obtained from the electronic structure calculations as the second, third and fourth derivatives of the electronic potentital energy surface with respect to the normal coordinates $\left\{Q_{i}\right\} . V_{c}$ and $V_{W}$ are the Coriolis coupling and the Watson's mass term, respectively. In the present study we ignore these two terms, and calculate the thermodynamic functions of the non-rotating molecule.

\subsection{The state specific vibrational self consistent field theory}

The VSCF approximation ${ }^{28-32}$ is based on separable ansatz for the vibrational wave function. The vibrational wave function of a $D$ - mode system is parametrized as

$$
\Psi\left(Q_{1}, Q_{2}, \ldots Q_{D}\right)=\prod_{i=1}^{D} \phi_{n_{i}}^{i}\left(Q_{i}\right) .
$$

Here, the single mode functions $\phi_{n_{i}}^{i}$ are called the modals. Each modal is expanded in a basis (usually in orthogonal harmonic oscillator basis)

$$
\phi_{n}^{i}=\sum_{m} \chi_{m}^{i} C_{m n} .
$$

The modals are determined by minimizing the expectation value of the Hamiltonian with respect to the modals, subject to the constrains that the modals are orthonormal. ${ }^{27,29-32}$ The resulting VSCF equations can be cast in the form of eigenvalue problem for each mode.

$$
\left(h_{i}^{u s c f}-\varepsilon_{n}^{i}\right) \phi_{n_{i}}^{i}\left(Q_{i}\right)=0 .
$$

Here, the effective single particle Hamiltonian, $h_{i}^{u s c f}$ is given by

$$
h_{i}^{v s c f}=-\frac{1}{2} \frac{d^{2}}{d Q_{i}^{2}}+\sum_{m=1}^{4} u_{i}^{(m)} Q_{i}^{m} .
$$

The coefficients $u_{i}^{(m)}$ reflect the average potential felt by the $i$ th mode. They are given by,

$$
\begin{aligned}
& u_{i}^{(1)}= \sum_{j<k} f_{i j k}\left\langle Q_{j}\right\rangle\left\langle Q_{k}\right\rangle+\sum_{j} f_{i j j}\left\langle Q_{j}^{2}\right\rangle \\
&+\sum_{j<k<l} f_{i j k l}\left\langle Q_{j}\right\rangle\left\langle Q_{k}\right\rangle\left\langle Q_{l}\right\rangle \\
&+\sum_{j<k} f_{i j j k}\left\langle Q_{j}^{2}\right\rangle\left\langle Q_{k}\right\rangle \\
&+\sum_{j} f_{i j j j}\left\langle Q_{j}^{3}\right\rangle, \\
& u_{i}^{(2)}=f_{i i}+\sum_{j} f_{i i j}\left\langle Q_{j}\right\rangle \\
&+\sum_{j} f_{i i j j}\left\langle Q_{j}^{2}\right\rangle \\
&+\sum_{j<k} f_{i i j k}\left\langle Q_{j}\right\rangle\left\langle Q_{k}\right\rangle, \\
& u_{i}^{(3)}=f_{i i i}+\sum_{j} f_{i i i j}\left\langle Q_{j}\right\rangle, \\
& u_{i}^{(4)}=f_{i i i i} .
\end{aligned}
$$

Starting with a set of guess basis functions, the SCF potential $u_{i}^{(m)}$ for a particular vibrational state is calculated. Equation (9) is then solved to obtain the modal energies and a new set of modal functions. This improved set of modal functions is then used to calculate the average SCF potential. This procedure is continued until the modal functions are converged.

In the ss-VSCF ${ }^{27}$ thermal calculations, a set of $B^{D}$ such VSCF calculations are performed to obtain the energies of $B^{D}$ vibrational states. Once the energies of all vibrational states are obtained, the partition function is obtained by the equation

$$
Z(T)=\sum_{n_{1} n_{2} \ldots} e^{\left.-\varepsilon\left(n_{1} n_{2} \ldots \ldots\right) / k_{B} T\right)} .
$$

Here, $n_{k}$ represents $n_{k}$ th eigenstate of the $k$ th mode. The other thermal properties are calculated using the standard statistical mechanical relations of the thermodynamic properties with the partition function. However, we note that a few aspects of ss-VSCF methods make it unattractive. First, the calculation of $Z(T)$ for a molecule with $D$ number of vibrational degrees of freedom requires $D$ nested do loops to obtain all the state energies. Thus, the computational cost increases exponentially with the number of vibrational degrees of freedom. This makes the ss-VSCF inaccessible for large 
molecules. Second, the nonlinear equation in Eq. (9) requires iterative solutions with the optimized potential for each state. Such iterative solutions sometimes do not converge for the potential of higher excited states, especially for large basis. Third, the underlying variational principle to obtain the VSCF equations ensures the upper bound of the ground state energy. However, the excitation energies in some occasion may fall below the exact value of the excitation energies due to variational collapse. ${ }^{36}$

\subsection{Virtual vibrational self-consistent field theory}

In the v-VSCF method, the VSCF Eq. (9) is solved for the vibrational ground state. This yields a set of modal functions $\phi_{n_{i}}^{i}\left(Q_{i}\right)$ and their energies $\varepsilon_{n}^{i}$ for each mode of vibration. The energy difference of a modal from the lowest energy modal of a particular mode is taken as the corresponding excitation energy. For example, excitation energy of a $k$ quanta excitation in the $i$ th vibrational mode is written as

$$
\Delta \varepsilon_{k}^{i}=\varepsilon_{k}^{i}-\varepsilon_{0}^{i} .
$$

The partition function for each mode is then

$$
z^{i}=e^{-\varepsilon_{0}^{i} / k_{B} T} \sum_{k} e^{\varepsilon_{k}^{i} / k_{B} T} .
$$

The total partition function of a molecule with $D$ vibrational modes is then given by

$$
Z=e^{-\left(V-V_{0}\right) / k_{B} T} \prod_{i=1}^{D} z^{i} .
$$

Thus, the partition function calculation requires only one do loop over the number of vibrational degrees of freedom and hence the computational effort to calculate the thermal properties scales linearly with the number of vibrational degrees of freedom.

\subsection{Thermal self-consistence field theory}

The formulation of t-SCF theory ${ }^{28}$ is based on the Gibbs-Bogoluibov inequality

$$
A \leq A_{0}+\left\langle H-H_{0}\right\rangle .
$$

Here, $A$ is the exact Helmholtz free energy defined as

$$
A=-k_{B} T \ln \left[\operatorname{Tr} e^{-H / k_{B} T}\right],
$$

and, $A_{0}$ is the Helmholtz free energy of a model system defined by a model Hamiltonian $H_{0}$. In the spirit of IPM, the model Hamiltonian is written as

$$
H_{0}=\sum_{k} h_{k}+E_{0},
$$

where, $h_{k}$ is an effective one particle Hamiltonian and $E_{0}$ is a temperature dependent constant. The form of $h_{k}$ is given by

$$
h_{k}=-\frac{1}{2} P_{k}^{2}+U_{k}\left(Q_{k}\right) .
$$

Here, $U_{k}\left(Q_{k}\right)$ is the one body effective potential obtained by minimizing the right hand side of Eq. (19). With a little algebraic manipulation one gets

$U_{k}\left(Q_{k}\right)=\left[\operatorname{Tr}_{l \neq k} V e^{-\sum_{l \neq k} h_{l} / k_{B} T}\right] /\left[\operatorname{Tr}_{l \neq k} e^{-\sum_{l \neq k} h_{l} / k_{B} T}\right]$.

With quartic approximation in the anharmonic potential such effective single particle potential takes the form

$$
U_{k}\left(Q_{k}\right)=u_{k}^{(1)} Q_{k}+u_{k}^{(2)} Q_{k}^{2}+u_{k}^{(3)} Q_{k}^{3}+u_{k}^{(4)} Q_{k}^{4} .
$$

The working equations for the parameters $u_{k}^{(m)}$ are obtained from Eq. (23).

$$
\begin{aligned}
u_{k}^{(1)}= & \sum_{i<j} f_{i j k}\left\langle Q_{i}\right\rangle_{T}\left\langle Q_{j}\right\rangle_{T}+\sum_{j} f_{k j j}\left\langle Q_{j}^{2}\right\rangle_{T} \\
& +\sum_{i<j<l} f_{i j k l}\left\langle Q_{i}\right\rangle_{T}\left\langle Q_{j}\right\rangle_{T}\left\langle Q_{l}\right\rangle_{T} \\
& +\sum_{i<j} f_{i j j k}\left\langle Q_{j}^{2}\right\rangle_{T}\left\langle Q_{i}\right\rangle_{T} \\
& +\sum_{j} f_{k j j j}\left\langle Q_{j}^{3}\right\rangle_{T},
\end{aligned}
$$

$$
\begin{gathered}
u_{k}^{(2)}=f_{k k}+\sum_{j} f_{k k j}\left\langle Q_{j}\right\rangle_{T}+\sum_{j} f_{k k j j}\left\langle Q_{j}^{2}\right\rangle_{T} \\
+\sum_{j<l} f_{k k j l}\left\langle Q_{j}\right\rangle_{T}\left\langle Q_{l}\right\rangle_{T}, \\
u_{k}^{(3)}=f_{k k k}+\sum_{j} f_{k k k j}\left\langle Q_{j}\right\rangle_{T}, \\
u_{k}^{(4)}=f_{k k k k} .
\end{gathered}
$$

Thus, the working equations for the parameters $u_{k}^{(m)}$ are similar of Eq. (11)-(14) with the replacement of quantum average of the normal coordinates $\left\langle Q_{k}^{m}\right\rangle$ by their thermal average counterpart $\left\langle Q_{k}^{m}\right\rangle_{T}$. The thermal $\left\langle Q_{k}^{m}\right\rangle_{T}$ are defined as

$$
\left\langle Q_{k}^{m}\right\rangle_{T}=\frac{\operatorname{Tr} Q_{k}^{m} e^{-h_{k} / k_{B} T}}{\operatorname{Tr} e^{-h_{k} / k_{B} T}} .
$$

Thus, the t-SCF equations are essentially the quantum mechanical VSCF equation generalized to finite temperature. Once the single parameters effective potentials are constructed, the eigenfunctions and eigenvalues of this single particle Hamiltonian $h_{k}$ are obtained by solving

$$
h_{k} \phi_{m}^{(k)}=\varepsilon_{m}^{(k)} \phi_{m}^{(k)}
$$


The eigenfunctions $\phi_{m}^{(k)}$ are the thermal analogs of the modal obtained in the VSCF theory. In practical implementation, this thermal modal are expanded in a suitable $\mathrm{HO}$ basis

$$
\phi_{n}^{(k)}=\sum_{m} \chi_{n}^{(k)} C_{m n}
$$

Substitution of this expansion in Eq. (30) generates matrix eigenvalue problem which is solved by standard numerical procedures. The thermal modals are then used to construct the thermal density matrices associated with each normal mode.

$$
\rho_{k}=e^{-h_{k} / k_{B} T}=\sum_{n}\left|\phi_{n}^{(k)}\right\rangle e^{-e_{n}^{(k)} / k_{B} T}\left\langle\phi_{n}^{(k)}\right| .
$$

These density matrices are then used to evaluate the thermal traces required to construct the potential in Eq. (23). Thus, the thermal trace in Eq. (29) is explicitly given by

$$
\left\langle Q_{k}^{m}\right\rangle_{T}=\frac{\sum_{m}\left\langle\phi_{m}^{(k)}\left|Q_{k}^{n}\right| \phi_{m}^{(k)}\right\rangle e^{-e_{n}^{(k)} / k_{B} T}}{\sum_{m} e^{-e_{n}^{(k)} / k_{B} T}} .
$$

Finally, the working equation for calculating the overall density matrix and the free energy are given by

$$
\begin{gathered}
\rho=\prod_{k} \rho_{k} e^{\left[-\left(V-V_{0}\right) / k_{B} T\right]}, \\
A=-k_{B} T \ln \left[\operatorname{Tr} e^{-\frac{H_{0}}{k_{B} T}}\right]+\left\langle V-V_{0}\right\rangle_{T} .
\end{gathered}
$$

Thus, the t-SCF procedure consists of the following steps. First, one starts with a set of guess modals and calculates associated free energy $A$, and the thermal density matrices $\rho_{k}$. In the next step, the single particle effective potential is calculated using these thermal density matrices. Equation (30) is then solved to obtain the modal energies and a new set of modals. The improved free energy and density matrices are calculated with this new set of modal. This process is iterated until either the free energy or the overall thermal density matrix is converged.

\section{Results and discussion}

We calculated the thermal averages of molecular properties of the $\mathrm{O}_{3}$ and $\mathrm{Cl}_{2} \mathrm{O}$ molecules using $\mathrm{v}-\mathrm{VSCF}$, ss-VSCF and t-SCF methods. In this study, we calculated the Helmholtz free energies, internal energies, entropies, expectation values of first normal coordinate $\left(\left\langle Q_{1}\right\rangle\right)$ and expectation values of the square of first normal coordinate $\left(\left\langle Q_{1}^{2}\right\rangle\right)$ of these two molecules over a wide range of temperatures starting from $10 \mathrm{~K}$ to $1000 \mathrm{~K}$. The results are compared with FVCI numbers. For $\mathrm{O}_{3}$, we use the quartic potential energy surface of
Lee and Susceria. ${ }^{37}$ We used Gaussian 09 package ${ }^{38}$ to generate the quartic potential energy surface of $\mathrm{Cl}_{2} \mathrm{O}$. It uses Barone algorithm ${ }^{39}$ for the quartic PES calculation. We used density functional theory-based method with B3LYP functional and aug-cc-pVTZ basis sets for this calculation. The geometry of this molecule was optimized tightly and ultra fine keyword was used as recommended by Barone. ${ }^{39}$ In all vibrational calculations, we used 6-12-6 harmonic oscillator basis functions. In all the thermal calculations, the scale of energy is taken such that the ground state energy is zero by the method used for the calculation.

We now discuss the results of Helmholtz free energy calculation by these three methods. We estimate the error in the free energy values calculated by these three methods from the FVCI values as

$$
\begin{aligned}
\Delta A= & {\left[A_{\mathrm{FVCI}}(T)-A_{\mathrm{FVCI}}(T=0)\right] } \\
& -\left[A_{a}(T)-A_{a}(T=0)\right] .
\end{aligned}
$$

We present, in figure 1, the variation of $\Delta A$ vs. temperature for the $\mathrm{Cl}_{2} \mathrm{O}$ and $\mathrm{O}_{3}$. In the low temperature region,
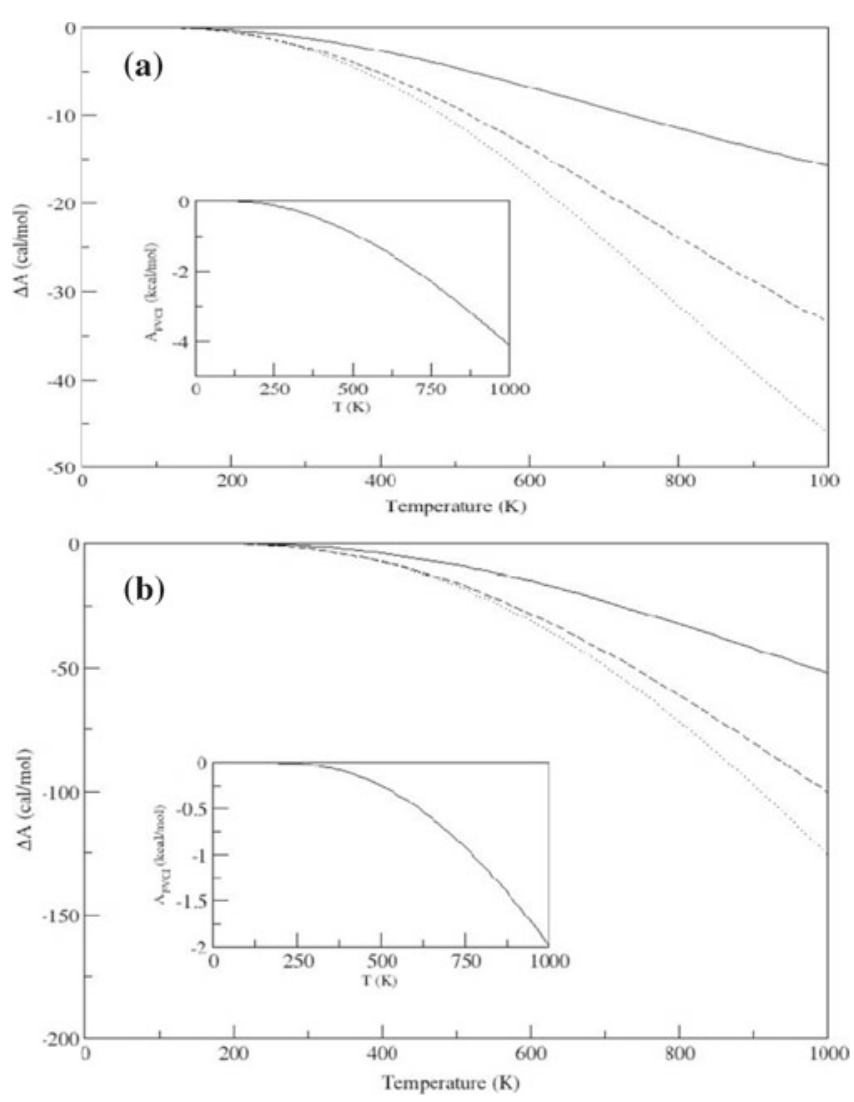

Figure 1. Variation of the error in the free energy ( $\triangle \mathrm{A}=$ $\left.\left[\mathrm{A}_{\mathrm{FVCI}}(\mathrm{T})-\mathrm{A}_{\mathrm{FVCI}}(\mathrm{T}=0)\right]-\left[\mathrm{A}_{\mathrm{a}}(\mathrm{T})-\mathrm{A}_{\mathrm{a}}(\mathrm{T}=0)\right]\right)$ with respect to temperature for ss-VSCF (full line), t-SCF (dashed line) and v-VSCF (dotted line). The inset is the FVCI free energy vs. temperature. (a) $\mathrm{Cl}_{2} \mathrm{O}$; (b) $\mathrm{O}_{3}$. 
all the three methods give similar results for both the molecules. The deviations in the free energy in these single particle based methods from FVCI are almost zero in this temperature region. The partition function at such low temperature is dominated by the Boltzmann factor of the ground state. The VSCF provides reasonably accurate description of the ground vibrational state. With increasing temperature we find deviations in the free energy by these three methods from FVCI values. The significant errors in the free energy by these three methods occur at lower temperature in $\mathrm{Cl}_{2} \mathrm{O}$ than $\mathrm{O}_{3}$. This is due to the presence of low frequency modes in $\mathrm{Cl}_{2} \mathrm{O}$, the higher energy states becomes populated in lower temperature in $\mathrm{Cl}_{2} \mathrm{O}$ than $\mathrm{O}_{3}$. However, the error in $\mathrm{O}_{3}$ is more than $\mathrm{Cl}_{2} \mathrm{O}$ due to stronger anharmonicity in $\mathrm{O}_{3}$. Among these three methods, we find that the ss-VSCF gives the most accurate results. At lower temperature, the v-VSCF and t-SCF give similar errors. However, as the temperature increases, the vVSCF becomes less accurate than t-SCF. For example, for the $\mathrm{O}_{3}$ molecule at $300 \mathrm{~K}$ temperature, the ss-VSCF gives about $4 \%$ error whereas the v-VSCF and t-SCF both give about $7 \%$ errors in the free energy values. At $500 \mathrm{~K}$ temperature, the ss-VSCF error in free energy is about $3 \%, \mathrm{v}$-VSCF error is $7 \%$ and t-SCF error is $6 \%$. At $1000 \mathrm{~K}$ temperature, we find that the error in the free energy calculations by ss-VSCF, v-VSCF and t-SCF methods are 3\%, 6\% and 5\%, respectively.

In figure 2, we present the variations of $\Delta \mathrm{U}$ of t-SCF, ss-VSCF and v-VSCF methods with respect to temperature for the $\mathrm{Cl}_{2} \mathrm{O}$ and $\mathrm{O}_{3}$ molecules, respectively. The error $\Delta \mathrm{U}$ is calculated using similar formula in Eq. (36). We find the deviations in the internal energies by these single particle models from the FVCI values at around $100 \mathrm{~K}$ temperature in case of $\mathrm{Cl}_{2} \mathrm{O}$. However, in case of $\mathrm{O}_{3}$, up to temperature around $200 \mathrm{~K}$; the single particle approximations give identical results of FVCI. Due to the presence of very low frequency modes in $\mathrm{Cl}_{2} \mathrm{O}$, the effect of correlations is observed in lower temperature compared to $\mathrm{O}_{3}$. Like the free energy values, here also, we find similar trend in the accuracy of internal energy by ss-VSCF, t-SCF and v-VSCF methods, the magnitude of error is ss-VSCF $<\mathrm{t}-\mathrm{SCF}<\mathrm{v}-\mathrm{VSCF}$. At lower temperatures, (for example, up to temperature $350 \mathrm{~K}$ in case of $\mathrm{O}_{3}$ ), the t-SCF and v-VSCF gives identical values of internal energy. However, as the temperature increases, the t-SCF performs better than v-VSCF. We find that for the $\mathrm{Cl}_{2} \mathrm{O}$ molecule, the errors in the internal energy increase up to a point and after that the error decreases with increasing temperature in all the three methods.

The next thermodynamic property we calculated is the entropy. In figure 3, we present the variation of the
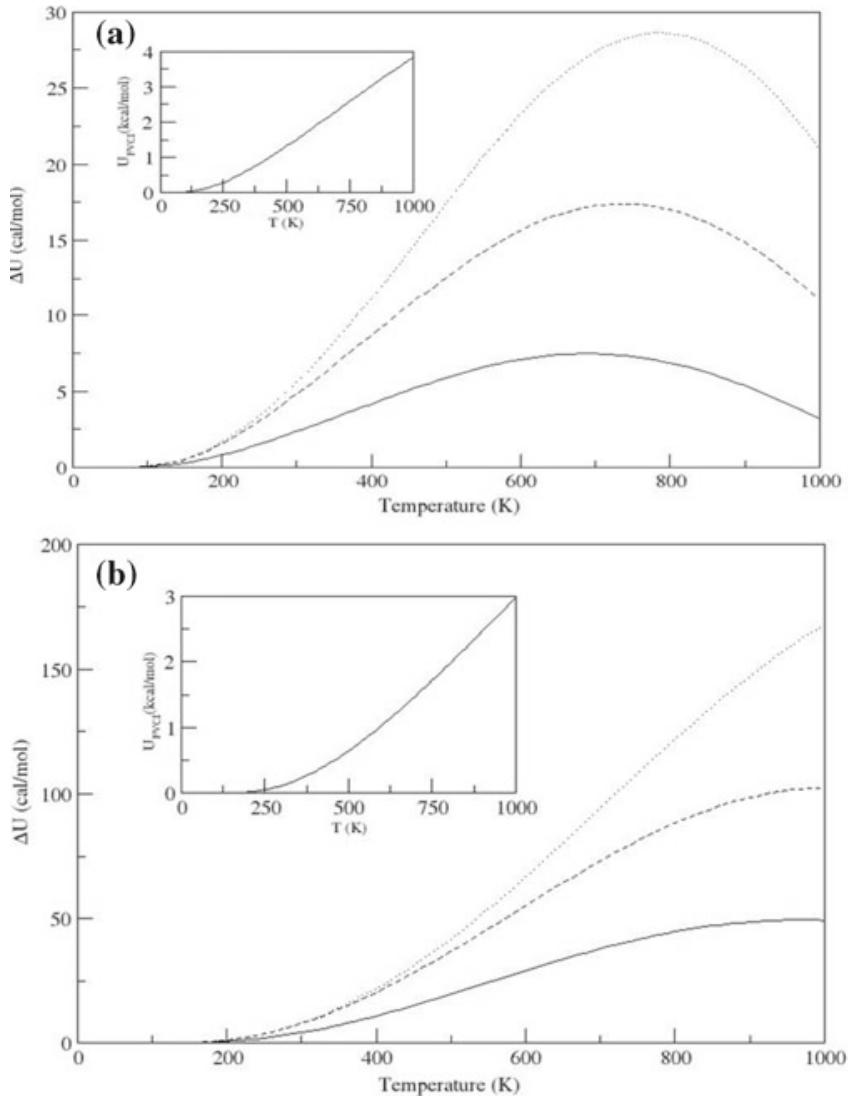

Figure 2. Variation of the error in the internal energy $\left(\Delta \mathrm{U}=\left[\mathrm{U}_{\mathrm{FVCI}}(\mathrm{T})-\mathrm{U}_{\mathrm{FVCI}}(\mathrm{T}=0)\right]-\left[\mathrm{U}_{\mathrm{a}}(\mathrm{T})-\mathrm{U}_{\mathrm{a}}(\mathrm{T}=0)\right]\right)$ with respect to temperature for ss-VSCF (full line), t-SCF (dashed line) and $\mathrm{v}-\mathrm{VSCF}$ (dotted line). The inset is the FVCI internal energy vs. temperature. (a) $\mathrm{Cl}_{2} \mathrm{O}$; (b) $\mathrm{O}_{3}$.

deviation of entropy values from FVCI with the variation of temperature for $\mathrm{Cl}_{2} \mathrm{O}$ and $\mathrm{O}_{3}$, respectively. Like the free energy and internal energy, in this case also, we find the trend in error is ss-VSCF $<\mathrm{t}-\mathrm{SCF}<\mathrm{v}-\mathrm{VSCF}$. The correlation effects are observed at lower temperature in case of $\mathrm{Cl}_{2} \mathrm{O}$ than $\mathrm{O}_{3}$. However, in all these three methods, the errors are more in case of $\mathrm{O}_{3}$ than $\mathrm{Cl}_{2} \mathrm{O}$ in all the temperature where the error is visible. For example, at $500 \mathrm{~K}$ temperature, the $\Delta \mathrm{S}$ value by ss-VSCF is $0.057 \mathrm{cal} \mathrm{K}^{-1} \mathrm{~mol}^{-1}$ in $\mathrm{O}_{3}$, whereas in $\mathrm{Cl}_{2} \mathrm{O}, \Delta \mathrm{S}$ value by the ss-VSCF is only $0.021 \mathrm{cal} \mathrm{K}^{-1} \mathrm{~mol}^{-1}$.

Like free energy and internal energy, in case of entropy also, the t-SCF and the v-VSCF methods give nearly identical values at lower temperature. However, as the temperature increases, the t-SCF method performs better than v-VSCF. Like the internal energy, the errors in entropy values by the independent particle models increase with the increasing temperature up to a point and the errors start falling after this temperature for the $\mathrm{Cl}_{2} \mathrm{O}$ molecule.

Next, we turn to the calculation of the expectation values of first normal coordinate $\left(\left\langle Q_{1}\right\rangle\right)$. In figure 4 , 

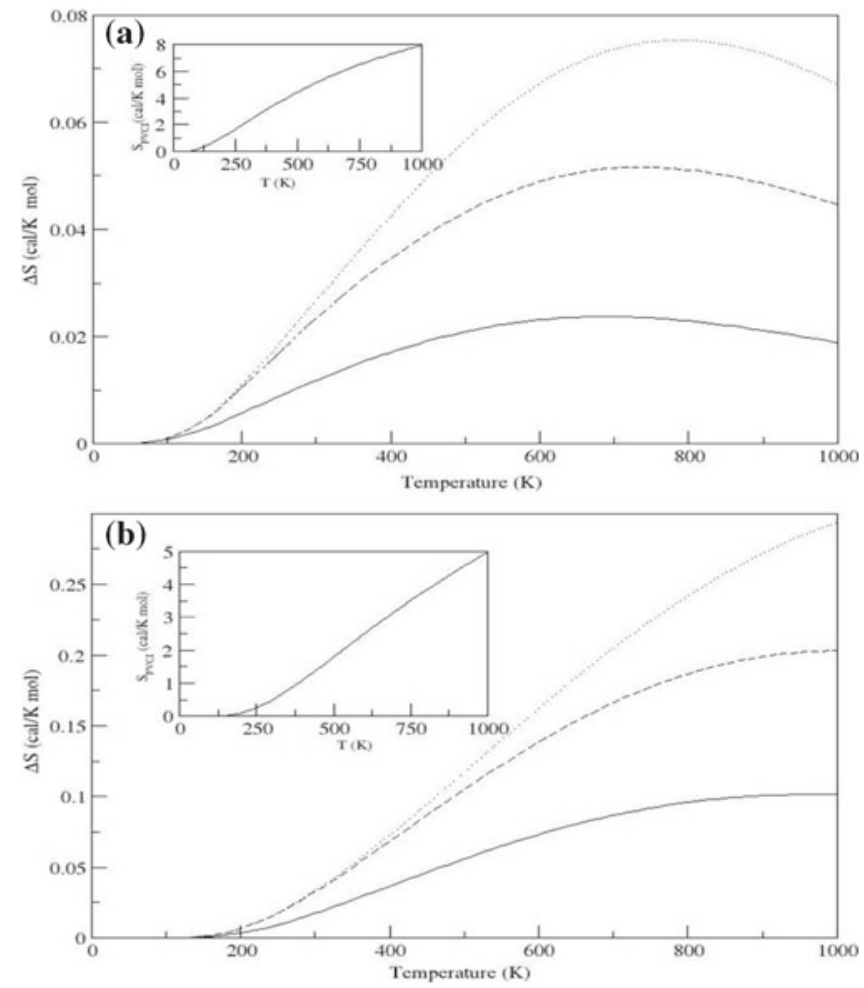

Figure 3. Variation of the error in the entropy ( $\Delta \mathrm{S}=$ $\left.\left[\mathrm{S}_{\mathrm{FVCI}}(\mathrm{T})-\mathrm{S}_{\mathrm{FVCI}}(\mathrm{T}=0)\right]-\left[\mathrm{S}_{\mathrm{a}}(\mathrm{T})-\mathrm{S}_{\mathrm{a}}(\mathrm{T}=0)\right]\right)$ with respect to temperature for ss-VSCF (full line), t-SCF (dashed line) and $\mathrm{v}-\mathrm{VSCF}$ (dotted line). The inset is the FVCI entropy vs. temperature. (a) $\mathrm{Cl}_{2} \mathrm{O}$; (b) $\mathrm{O}_{3}$.

we present the variation of $\left\langle Q_{1}\right\rangle$ (defined as $\left\langle Q_{1}\right\rangle_{\mathrm{FVCI}}-$ $\left\langle Q_{1}\right\rangle_{a}$ ) with temperature for $\mathrm{Cl}_{2} \mathrm{O}$ and $\mathrm{O}_{3}$, respectively. Here also, we find similar trend in the accuracy of these three methods, i.e., the ss-VSCF gives most accurate $\left\langle Q_{1}\right\rangle$ value followed by t-SCF and the v-VSCF is the least accurate one. We find that the t-SCF accuracy for $\left\langle Q_{1}\right\rangle$ is significantly higher than the v-VSCF counterpart, especially at higher temperatures. The error in the calculated $\left\langle Q_{1}\right\rangle$ value increases with increasing temperature. For example, in $\mathrm{O}_{3}$ molecule, at $300 \mathrm{~K}$ temperature, the error in ss-VSCF calculation is $2 \%$. The errors in this temperature by the t-SCF and the v-VSCF methods are $2 \%$ and $4 \%$, respectively. The maximum error in $\left\langle Q_{1}\right\rangle$ for this molecule in this temperature range by ss-VSCF method is $5 \%$ at $1000 \mathrm{~K}$ temperature. The $\mathrm{t}-$ $\mathrm{SCF}$ calculation at this temperature gives error in $\left\langle Q_{1}\right\rangle$ $9 \%$ whereas the error by the $\mathrm{V}$-VSCF method is as high as $35 \%$. In case of $\mathrm{Cl}_{2} \mathrm{O}$ also, the t-SCF and the ssVSCF methods give similar accuracy in the $\left\langle Q_{1}\right\rangle$ calculation which is far better than the v-VSCF method especially at higher temperature. For example, at $1000 \mathrm{~K}$ temperature, the error in ss-VSCF calculation is about $1 \%$ and that in t-SCF calculation is about $2 \%$. In case of $\mathrm{v}$-VSCF calculation the error in $\left\langle Q_{1}\right\rangle$ is $37 \%$. The
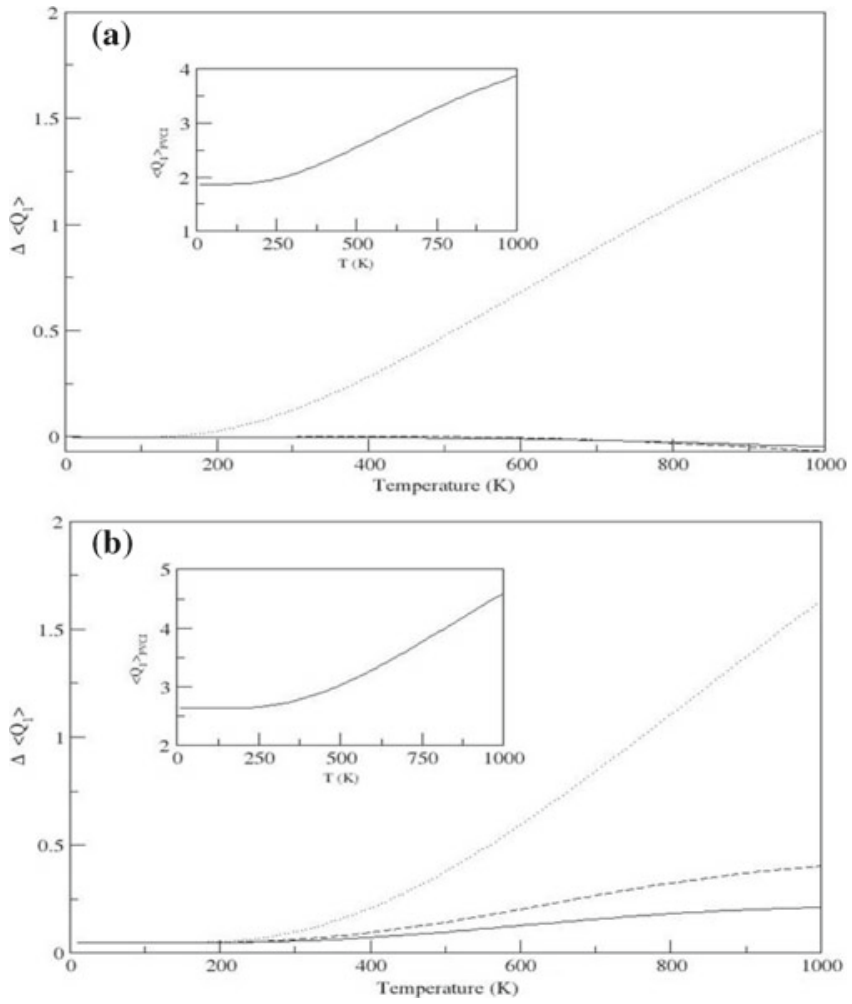

Figure 4. Variation of the error in the expectation values of $Q$ for first mode $\left(\Delta\left\langle Q_{1}\right\rangle=\left\langle Q_{1}\right\rangle_{\mathrm{FVCI}}-\left\langle Q_{1}\right\rangle_{\mathrm{a}}\right)$ with respect to temperature for ss-VSCF (full line), t-SCF (dashed line) and v-VSCF (dotted line). The inset is the FVCI $\left\langle Q_{1}\right\rangle$ values vs. temperature. (a) $\mathrm{Cl}_{2} \mathrm{O}$; (b) $\mathrm{O}_{3}$.

results show that the t-SCF accuracy is comparable with the ss-VSCF accuracy. However, in comparison to the $\mathrm{v}-\mathrm{VSCF}$, the t-SCF gives far better results in the $\left\langle Q_{1}\right\rangle$ calculation.

A similar trend is seen in the case of $\left\langle Q_{1}^{2}\right\rangle$ calculation. In figure 5, we plot the variation of $\left\langle Q_{1}^{2}\right\rangle$ with respect to temperature for $\mathrm{Cl}_{2} \mathrm{O}$ and $\mathrm{O}_{3}$ molecule, respectively. Like the other properties, here also we find the error trend is ss-VSCF $<\mathrm{t}-\mathrm{SCF}<\mathrm{v}-\mathrm{VSCF}$. The error increases with the increase of temperature for all the three methods. As can be seen from the graph that the all the three methods give similar accuracy in $\left\langle Q_{1}^{2}\right\rangle$ at lower temperature region. Like $\left\langle Q_{1}\right\rangle$, in case of $\left\langle Q_{1}^{2}\right\rangle$ also, we find that the t-SCF performs much better than the v-VSCF counterpart. For example, at $700 \mathrm{~K}$ temperature, the t-SCF gives $7 \%$ error in the $\left\langle Q_{1}^{2}\right\rangle$ value compared $4 \%$ error in the ss-VSCF calculation. The vVSCF error in this case is as high as $9 \%$. At $1000 \mathrm{~K}$ temperature, the t-SCF error is $9 \%$ compared to $5 \%$ in ss-VSCF. However, with the v-VSCF calculations the error is $15 \%$.

Finally, a few comments on the computational resource needed for these calculations are in order. Although the ss-VSCF method is found to be the 

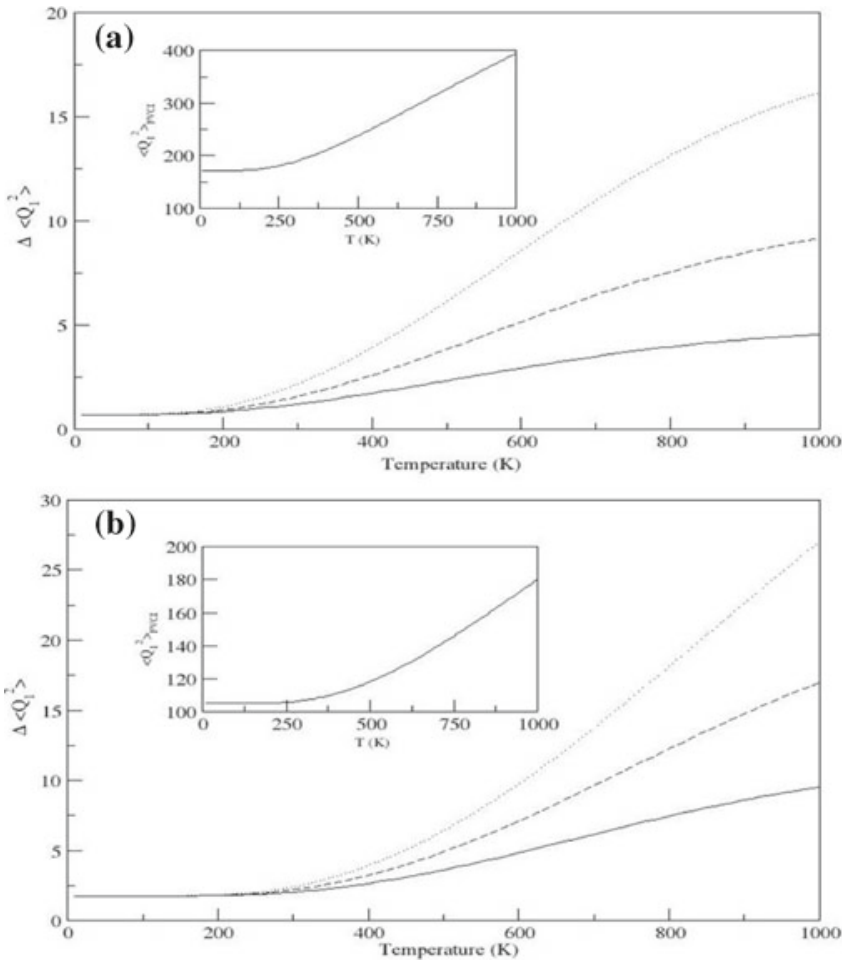

Figure 5. Variation of the error in the expectation values of $Q$ for first mode $\left(\Delta\left\langle Q_{1}^{2}\right\rangle=\left\langle Q_{1}^{2}\right\rangle_{\mathrm{FVCI}}-\left\langle Q_{1}^{2}\right\rangle_{\mathrm{a}}\right)$ with respect to temperature for ss-VSCF (full line), t-SCF (dashed line) and v-VSCF (dotted line). The inset is the FVCI $\left\langle Q_{1}^{2}\right\rangle$ values vs. temperature. (a) $\mathrm{Cl}_{2} \mathrm{O}$; (b) $\mathrm{O}_{3}$.

most accurate method among these three single particle approximations to calculate the thermal averages of the molecular properties, it becomes computationally very expensive for even moderate size molecule. The number of states scales as $B^{D}$, where $B$ is the number of basis functions per degrees of freedom and $D$ is the number of vibrational degrees of freedom. The computational scaling for the VSCF calculation of each state goes as follows. The SCF Hamiltonian construction goes as $B^{2} D$ and the matrix diagonalization goes as $B^{3} D$. Thus, the ss-VSCF method becomes resource hungry for large molecules. The t-SCF requires one SCF calculation for each temperature. The SCF procedure requires the construction of the temperature dependent hamiltonian that scales as $D B^{2}$ and its diagonalization $\left(O\left(D B^{3}\right)\right)$. The calculation of $\left\langle V-V_{0}\right\rangle_{T}$ scales as $T D^{4}$, where, $T$ is the number of temperature steps. Lastly, the v-VSCF requires only one VSCF calculation. So it requires an effort proportional to $T D$.

\section{Conclusion}

In this work, we presented a comparative study of the three IPM based approximations, ss-VSCF, t-SCF and $\mathrm{v}$-VSCF to calculate the thermal averages of mole- cular properties. With the illustrative examples of the $\mathrm{Cl}_{2} \mathrm{O}$ and $\mathrm{O}_{3}$ molecules, we find that the IPM based model provides reasonably accurate results in the thermal averages calculations compared to the FVCI numbers. At lower temperature domain, the results obtained by these IPM based methods are very close to the FVCI values. However, with increasing the temperature, we find deviations in the thermal average values by IPM based methods from the FVCI results increase.

Among these three IPM based methods, we find that the ss-VSCF method provides the most accurate results followed by t-SCF and v-VSCF method. Even at higher temperatures, the ss-VSCF results are comparable to the FVCI values. The ss-VSCF method, however, suffers from few inherent drawbacks. Since the ss-VSCF involves the VSCF calculations of all the vibrational states within the basis set used, it becomes computationally very expensive for large molecules. Second, the ss-VSCF method uses optimized potential for each state. The optimized potentials for higher excited states do not always lead to a converged solution for the VSCF equations. This leads to difficulties in carrying out the ss-VSCF calculation even for triatomic molecules. For example, in $\mathrm{O}_{3}$ molecule, the VSCF wave function fails to converge for $1_{5} 2_{2} 3_{7}$ state when 8-16-8 $\mathrm{HO}$ basis functions are used in the calculation. Third, as stated earlier, the ss-VSCF free energy is not guaranteed to be the upper bound due to possibility of the variational collapse in the excitation energies. Lastly, we note that the VSCF wave functions are not orthogonal, since they are eigenfunctions of different average potentials. As a consequence, it becomes difficult to write down the density matrices in terms of these functions without making further approximations to the overlap matrix between these functions.

In case of t-SCF, the computational cost of t-SCF method is much less than the ss-VSCF method. Thus, the t-SCF calculations can be easily carried out for the thermal averages of large molecules. ${ }^{28}$ The t-SCF has another important feature. Since it uses the Feynman variation principle based on the Gibbs-Bogoliubov inequality to calculate the free energy, it guarantees the upper bound to the free energy. Thus, as pointed by Roy and Prasad, ${ }^{28}$ the t-SCF due to the upper bound nature of the calculated free energy, should be a method of choice when one calculates the difference in energies e.g., the equilibrium constant calculation. The errors by the t-SCF methods in that case are in the same direction for both the reactant and product molecules and thus they tend to cancel each other, at least partially. In terms of accuracy in the calculated thermal average values, the t-SCF gives comparable numbers to ss-VSCF values with much less computational cost. Moreover, unlike 
the ss-VSCF method, the t-SCF does not suffer from the convergence problem. The t-SCF uses single particle potentials generated by thermally averaged many body potential to construct the effective single particle Hamiltonian. The thermally averaged potential is dominated by the ground state potentials due to the large Boltzmann factor of that state. The convergence problem in the solution of VSCF equations with the ground state potential is very unlikely. Moreover, the present studies indicate that the density matrix is better represented by the t-SCF approach. This is apparent from the near identical results by ss-VSCF and t-SCF for the expectation values for $Q$ and $Q^{2}$.

Finally, the v-VSCF method offers very fast calculations of the thermal averages even for large molecules as the computational cost scales linearly with the number of vibrational degrees of freedom. Like the t-SCF method, since it involves the VSCF calculation only for the ground state, it does not suffer from the convergence problem. However, in terms of accuracy the v-VSCF performs poorly compared to the ss-VSCF and t-SCF, especially in the high temperature region. The density matrix by this approach appears to be particularly prone to errors, as seen from the results of the expectation values.

\section{Acknowledgements}

SB thanks the Dr. D S Kothari Post-Doctoral Fellowship from the University Grants Commission (UGC), India. MDP acknowledges support from UGC, in the form of CAS to the School of Chemistry, UPE thanks the University of Hyderabad and the Department of Science and Technology (DST), India for HPCF facility at the University of Hyderabad.

\section{References}

1. Njegic B and Gordon M S 2006 J. Chem. Phys. 125 224102

2. Barone V 2004 J. Chem. Phys. 1203059

3. Miekle S L and Truhlar D G 2009 J. Phys. Chem. A113 8717

4. Feynman R P and Kleinert H 1986 Phys. Rev. A34 5080

5. Palmieri B and Ronis D 2006 Phys. Rev. E73 061106

6. Zhao Y and Miller W H 2002 J. Chem. Phys. 1179605

7. Makri N and Miller W H 2002 J. Chem. Phys. 1169207

8. Korsch H J 1979 J. Phys. A12 1521

9. Messina M, Schenter G K and Garret G 1993 J. Chem. Phys. 1038303

10. Singer K and Smith W 1986 Mol. Phys. 57761

11. Hellsing B, Sawada S and Meitu H 1985 Chem. Phys. Lett. 122303

12. Rickman J M and LeSar R 2002 Ann. Rev. Matter. Res. 32195
13. Sanyal G, Mandal S H and Mukherjee D 1992 Chem. Phys. Lett. 19255

14. Sanyal G, Mandal S H, Guha S and Mukherjee D 1993 Phys. Rev. E48 3373

15. Roy T K and Durga Prasad M 2011 J. Chem. Phys. 134 214110

16. Frantsuzov P, Neumaier A and Mandelshtam V A 2003 Chem. Phys. Lett. 381117

17. Frantsuzov P and Mandelshtam V A 2004 J. Chem. Phys. 1219247

18. Shao J and Pollak E 2006 J. Chem. Phys. 125133502

19. Isaacson A D and Truhlar D G 1981 J. Chem. Phys. 75 4090

20. Chakrabarty A, Truhlar D G, Bowman J M and Carter S 2004 J. Chem. Phys. 1212071

21. Chakrabarty A and Truhlar D G 2006 J. Chem. Phys. 124184310

22. Truhlar D G and Isaacson A D 1991 J. Chem. Phys. 94 357

23. Isaacson A D and Zhang X 1988 Theor. Chim. Acta 74 493

24. Isaacson A D and Hung S C 1994 J. Chem. Phys. 101 3928

25. Isaacson A D 1998 J. Chem. Phys. 1089978

26. Kongsted J and Christiansen O 2007 J. Chem. Phys. 127 154315

27. Hansen M B, Christiansen O, Toffoli D and Kongsted J 2008 J. Chem. Phys. 128174106

28. Roy T K and Durga Prasad M 2009 J. Chem. Phys. 131 114102

29. Carney D C, Sprandel L L and Kern C W $1978 A d v$. Chem. Phys. 37305

30. Bowman J M 1978 J. Chem. Phys. 68608

31. Bowman J M 1986 Acc. Chem. Res. 19202

32. Christoffel K M and Bowman J M 1985 Chem. Phys. Lett. 85220

33. Ratner M A and Gerber R B 1986 J. Phys. Chem. 9020

34. Feynman R P 1974 Statistical mechanics (Reading, MA: Benjamin)

35. Watson J K G 1968 Mol. Phys. 15479

36. Epstein S T 1974 Variation method in quantum chemistry (New York: Academic Press)

37. Lee T J and Scuseria G E 1990 J. Chem. Phys. 93489

38. Frisch M J, Trucks G W, Schlegel H B, Scuseria G E, Robb M A, Cheeseman J R, Scalmani G, Barone V, Mennucci B, Petersson G A, Nakatsuji H, Caricato M, Li X, Hratchian H P, Izmaylov A F, Bloino J, Zheng G, Sonnenberg J L, Hada M, Ehara M, Toyota K, Fukuda R, Hasegawa J, Ishida M, Nakajima T, Honda Y, Kitao O, Nakai H, Vreven T, Montgomery Jr. J A, Peralta J E, Ogliaro F, Bearpark M, Heyd J J, Brothers E, Kudin K N, Staroverov V N, Kobayashi R, Normand J, Raghavachari K, Rendell A, Burant J C, Iyengar S S, Tomasi J, Cossi M, Rega N, Millam J M, Klene M, Knox J E, Cross J B, Bakken V, Adamo C, Jaramillo J, Gomperts R, Stratmann R E, Yazyev O, Austin A J, Cammi R, Pomelli C, Ochterski J W, Martin R L, Morokuma K, Zakrzewski V G, Voth G A, Salvador P, Dannenberg J J, Dapprich S, Daniels A D, Farkas O, Foresman J B, Ortiz J V, Cioslowski J, Fox D J 2009 Gaussian 09, Revision A.1 (Wallingford, CT: Gaussian, Inc.)

39. Barone V 2005 J. Chem. Phys. 122014108 